\title{
5 Research Square

\section{The Impact of Daily Urinary Volume Variations and Prostate Volume on Prostate Position During Radiotherapy}

\section{Kentaro Nishioka}

Hokkaido University https://orcid.org/0000-0002-8272-7603

Takayuki Hashimoto ( $\nabla$ thashimoto@med.hokudai.ac.jp )

Hokkaido University

Takashige Abe

Hokkaido University

Takahiro Osawa

Hokkaido University

Ryuji Matsumoto

Hokkaido University

Isao Yokota

Hokkaido University

Norio Katoh

Hokkaido University

Rumiko Kinoshita

Hokkaido University

Koichi Yasuda

Hokkaido University

Toshiaki Yakabe

Hokkaido University

Takaaki Yoshimura

Hokkaido University

Seishin Takao

Hokkaido University

Nobuo Shinohara

Hokkaido University

Hidefumi Aoyama

Hokkaido University

Shinichi Shimizu

Hokkaido University 
Research

Keywords: prostate cancer, proton therapy, urinary volume, prostate movement

Posted Date: December 8th, 2020

DOl: https://doi.org/10.21203/rs.3.rs-120617/v1

License: (c) (i) This work is licensed under a Creative Commons Attribution 4.0 International License. Read Full License 


\section{Abstract}

\section{Background}

Daily variations in bladder volumes are thought to be a major cause of prostate position changes during radiotherapy, however, the impact of such changes on prostate position is not fully established. We hypothesized that prostate volume (PV) could also be related to the susceptibility of prostate movement to changes in urinary volume (UV). The purpose of this study is to investigate the impact of daily UV variations and $\mathrm{PV}$ on the prostate position during radiotherapy.

\section{Methods}

A total of 268 treatment sessions in 12 consecutive prostate cancer patients who were treated with proton beam therapy in our hospital were retrospectively analyzed. All patients were instructed to void urine and feces one hour before the treatment and the UV was measured by a portable ultrasound device just before the treatment. After the positional registration based on bone structure, the prostate positional difference from the planned position was measured using fiducial markers in the prostate and a real-time tumor tracking system. The measurements were for the differences in the left-right (LR), cranial-caudal (CC), and anterior-posterior (AP) directions. The PV was measured by ultrasound imaging before every treatment. The impact of the UV variations on the prostate position was evaluated by the linear mixed effect model, and including PV $(<20 \mathrm{ml}$ and $\geq 20 \mathrm{ml})$ as covariates.

\section{Results}

There was significant movement of the prostate in the posterior direction with increasing UV in patients with $\mathrm{PV} \geq 20 \mathrm{ml}$ ( $p=0.04$, estimated coefficient of regression $-0.436 \mathrm{~mm} / 100 \mathrm{ml}, 95 \% \mathrm{Cl}$ from -0.854 to -0.018). However, there was no significant correlation between UV changes and movement of the prostate in either of the LR, CC, or AP directions in all cases and patients with $\mathrm{PV}<20 \mathrm{ml}$.

\section{Conclusions}

Our results suggest that the increase of UV could result in posterior prostate movement in patients with prostatic hyperplasia, however, the clinical impact is thought to be small. There is however a need for further research to identify other factors that could affect the daily changes in prostate position as well as a need for a more ideal preparation protocol to improve the reproducibility of the treatment-planning CT.

\section{Background}

Radiotherapy is a major curative treatment for early prostate cancer. Since the radiotherapy is carried out based on images that were acquired before the treatment, in the present study treatment planning computed tomography (RT-CT), there is a need to ensure that the difference of the prostate position between the treatment plan and the actual position is minimal. Recent advances in image guidance 
techniques have made it possible to know changes in the position of target organs in the body. However, dose distributions could be affected by changes in the target position, especially in proton therapy. Therefore, the reproducibility of the prostate position between the RT-CT and the actual treatment is still important in the setting of proton therapy[1-3].

The bladder is one of the major organs at risk (OARs) in prostate radiotherapy. Previous studies that evaluated the effect of bladder filling on dose-volume distributions in prostate radiotherapy reported that an increase in the doses to the bladder and bowel loops occurred as a result of treatment plans with empty bladders $[4,5]$. A recent survey in the United Kingdom reported that the majority $(98 \%)$ of departments used a bladder filling protocol[6].

One of the main factors in the changes of the position of the prostate during radiotherapy is the change in urinary volume (UV) in the bladder[7-12]. Considerations to keep UV constant during radiotherapy are widely recommended[6,13-16]. However, the amount of urine accumulated within a certain period of time varies widely among individuals, and the fluctuations are large despite pretreatment preparation[17]. In addition, the impact of UV changes on prostate position during radiotherapy is not commonly agreed on. Some studies have concluded that the UV changes during treatment correlated with the displacement of the prostate[7-12], while others found no such correlation[4, 17-19]. We hypothesized that the prostate volume (PV) could also be related to the susceptibility of prostate movement due to changes in UV, however, there is no report of details of this relationship.

It has been difficult to measure daily UV and the amount of prostate movement at each time during radiotherapy. Presently however, the placement of fiducial markers within the prostate and use of realtime tumor tracking enables an accurate determination of how far the prostate has moved from a planned position relative to bony structures during each treatment. In addition, ultrasound equipment specialized for measuring UV in the bladder has come into common use, and it has become possible to measure UV in each radiotherapy treatment session. In this study, we investigated the impact of daily UV variations and $\mathrm{PV}$ on the prostate position during radiotherapy.

\section{Methods}

\section{Patients}

A total of 268 treatment sessions in 12 consecutive prostate cancer patients who were treated with proton beam therapy in our hospital were retrospectively analyzed. This study was approved by the Institutional Review Board of Hokkaido University Hospital for Clinical Research (016-0210).

\section{Treatment preparation protocol}

The RT-CT images with a slice thickness of $1.25 \mathrm{~mm}$ were acquired for all patients, approximately one week after the fiducial marker implant. A vacuum cushion was used to set the patient's body. One hour before the RT-CT, patients were orally instructed to void as much urine as possible. There were no 
restrictions on diet or drinking of water. When residual stool or gas was observed in the rectum, we evacuated this using a catheter or by patients themselves prior to image acquisition. Similarly, at the treatment, urination and defecation were instructed to take place one hour before the treatment. If residual feces and/or gas were observed in the rectum, the treatment was carried out after evacuation. Treatment consisted of 30 fractions to the prostate with or without including the seminal vesicles.

\section{Urinary volume (UV) measurements}

The baseline UV was defined as that obtained from RT-CT images which was calculated by contouring the water dense area of the bladder.

The UV was measured three times with an ultrasound device (Bladder scan BVI9400, Verathon) just before the initiation of the treatment, and the mean of these was defined as the UV at the time of treatment. The nominal accuracy of the device is $\pm 15 \%$. Previous studies suggest strong correlations between the volumes measured by bladder scan and the CT contoured volume in prostate radiotherapy patients[17, 20], however, bladder scan findings are reported to be unreliable in obese and ascites patients[21], and we determined obesity by body mass index (BMI) and ascites by RT-CT. All UV measurements were performed by the same person.

\section{Prostate volume (PV) measurements}

The PV was measured using transrectal ultrasound images assuming a prolate ellipsoid shape: multiplying the largest anteroposterior (height, $H$ ), transverse (width, $W$ ) and craniocaudal (length, $L$ ) prostate diameters(centimeters) by $\pi / 6(H \times W \times L \times \pi / 6(\mathrm{ml}))$. The measurements were performed by experienced urologists before all treatments for prostate cancer.

\section{Prostate position measurements}

Three or four spherical fiducial markers were implanted percutaneously in the prostatic gland about a week prior to the RT-CT. At first, registration was performed using two $\mathrm{kV} \mathrm{X}$ ray images of the anterior and lateral side with reference to the bone structure. Then, the distance from the planned position to the actual position of the markers, the prostate movement relative to the bone structure were measured in the Left-Right (LR), Cranial-Caudal (CC) and Anterior-Posterior (AP) directions using a real-time tumor tracking system. The details of this method was reported in our previous report[22].

\section{Analysis of correlation between UV changes and prostate movement}

The amount of changes in UV was defined as the UV at the time of treatment minus the baseline UV. The correlation between the UV change and prostate movement were assessed using linear mixed-effects models including PV ( $<20 \mathrm{ml}$ and $\geqq 20 \mathrm{ml})$ as covariates. The PV threshold $(20 \mathrm{ml})$ was set based on clinical guidelines for male lower urinary tract symptoms and benign prostatic hyperplasia[23]. The level of significance was set at $p=0.05$ or less, and the clinical impact was assessed using the $95 \%$ 
confidence intervals $(\mathrm{Cl})$. The statistical analysis was performed using JMP Pro (ver. 14, SAS institute, Cary, NC, USA).

\section{Results}

Details of patient characteristics are shown in Table 1. The number of measurements in a patient was between 8 and 30 (median 27). There were two obese patients (BMI 27.7 and 35.1) and none of the other patients exceeded BMI 25. There were no significant differences in the BMI of patients with $P V<20 \mathrm{ml}$ and PV $\geq 20 \mathrm{ml}$ ( $p=0.54$, Welch's $t$ test). None of the patients had significant ascites on the RT-CT images. 
Table 1

Patient characteristics

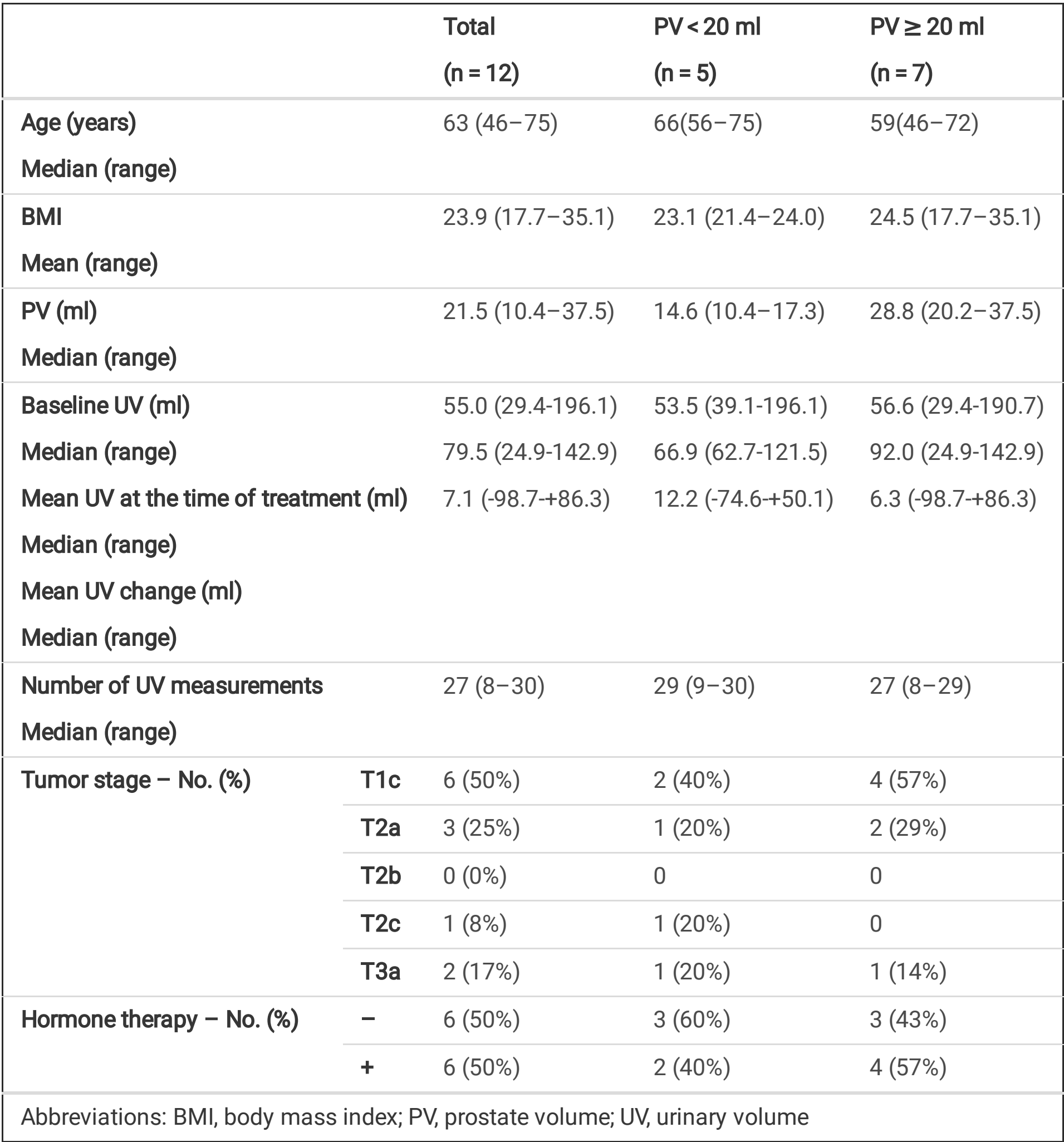

The UV in the patients on the RT-CT images was from 29.4 to $196.1 \mathrm{ml}$ (median $55.0 \mathrm{ml}$, standard deviation $58.8 \mathrm{ml}$ ) and the mean UV in the patients during actual treatment was from 24.9 to $142.9 \mathrm{ml}$ (median $79.5 \mathrm{ml}$, standard deviation $37.8 \mathrm{ml}$ ). The mean UV change in the patients was from -98.7 to $86.3 \mathrm{ml}$ (median $7.1 \mathrm{ml}$, standard deviation $51.7 \mathrm{ml}$ ). The mean prostate movement (standard deviation range) from the RT-CT position was from -1.46 to $1.85 \mathrm{~mm}(0.21$ to $0.60 \mathrm{~mm}),-6.10$ to $3.65 \mathrm{~mm}$ ( 0.77 to $2.52 \mathrm{~mm})$, and -1.90 to $5.23 \mathrm{~mm}(0.58$ to $2.19 \mathrm{~mm})$ in the LR, CC, and AP directions, respectively. The 
positive values indicate that the prostate moved to the left, cranial, and anterior directions in a patient, respectively.

The linear mixed effect model showed no significant relationship between the UV changes and prostate movement in any direction, except for patients with $P V \geq 20 \mathrm{ml}$ in the AP direction $(p=0.04)$ (Fig. 1, bottom right panel). The detailed results can be found in Table 2 . The estimated coefficient of regression of the PV $\geq 20 \mathrm{ml}$ patient group in the AP direction was $-0.436 \mathrm{~mm} / 100 \mathrm{ml}(95 \% \mathrm{Cl}$ was from -0.854 to $-0.018 \mathrm{~mm} / 100 \mathrm{ml}$ ). Expressed differently, the prostate may move up to only $0.854 \mathrm{~mm}$ posteriorly when UV increases by $100 \mathrm{ml}$. Figure 2 shows an example of two sagittal CT images for the same patient with $\mathrm{PV}=23.5 \mathrm{ml}$ obtained at different times. This image indicates that the position of the fiducial marker in the prostate was very nearly unchanged despite the large UV change. Figure 1 (panels in the middle and right columns) also demonstrates a large prostate position variation in the $\mathrm{CC}$ and AP directions between patients also with small UV changes.

Table 2

The estimated coefficient of regression and $95 \%$ confidence interval derived from the linear mixed effect model

\begin{tabular}{|c|c|c|c|c|c|c|c|c|c|}
\hline & \multicolumn{3}{|c|}{ Left-Right } & \multicolumn{3}{|c|}{ Cranial-Caudal } & \multicolumn{3}{|c|}{ Anterior-Posterior } \\
\hline & E & $\mathrm{p}$ & $95 \% \mathrm{Cl}$ & $E$ & $\mathrm{p}$ & $95 \% \mathrm{Cl}$ & E & $\mathrm{p}$ & $95 \% \mathrm{Cl}$ \\
\hline Total & 0.007 & 0.88 & $\begin{array}{l}-0.086 \text { to } \\
0.099\end{array}$ & -0.072 & 0.65 & $\begin{array}{l}-0.386 \text { to } \\
0.243\end{array}$ & 0.140 & 0.31 & $\begin{array}{l}-0.134 \\
\text { to } \\
0.414\end{array}$ \\
\hline $\begin{array}{l}P V< \\
20 \mathrm{ml}\end{array}$ & 0.102 & 0.13 & $\begin{array}{l}-0.030 \text { to } \\
0.234\end{array}$ & -0.282 & 0.16 & $\begin{array}{l}-0.635 \text { to } \\
0.071\end{array}$ & -0.120 & 0.50 & $\begin{array}{l}-0.470 \\
\text { to } \\
0.231\end{array}$ \\
\hline $\begin{array}{l}P V \geq \\
20 \mathrm{ml}\end{array}$ & -0.103 & 0.12 & $\begin{array}{l}-0.233 \text { to } \\
0.028\end{array}$ & 0.165 & 0.52 & $\begin{array}{l}-0.346 \text { to } \\
0.677\end{array}$ & 0.436 & 0.04 & $\begin{array}{l}0.018 \\
\text { to } \\
0.854\end{array}$ \\
\hline
\end{tabular}

\section{Discussion}

Previous reports have suggested that the changes in bladder and rectum volume during radiotherapy caused a significant prostate positional shift, and based on that pre-treatment protocols such as bladder filling and bowel emptying have frequently been deployed[6-11, 13, 24]. However, other reports found no significant correlation between daily variations in bladder filling and random prostate motion[4, 17-19]. The previously reported correlation between UV changes and prostate movement was based on limited samples with comparisons of pre-treatment CT and CT images recorded after the initiation of the radiotherapy. Gurjar et al. reported about prostate movement and dosimetric variations because of 
bladder and rectum volume changes using daily cone-beam $\mathrm{CT}(\mathrm{CBCT})$. They concluded that bladder and rectum volume affects the position of the prostate, but no data was shown to substantiate the correlation[25]. This makes us believe that the correlation between UV changes and prostate movement is still not proven and that confounding factors such as PV could have affected the correlation.

Our results indicate a significant posterior movement of the prostate with increases in UV, which is consistent with previous reports, but the change was observed only in patients with PV $\geq 20 \mathrm{ml}$ (Fig. 1 bottom right panel). However, although the change was statistically significant, a movement of less than $1 \mathrm{~mm}$ can be expected to result from a $100 \mathrm{ml}$ increase in UV, and additionally, there are large variations in the prostate position changes between patients even if the UV changes were small. This indicates that the clinical significance of maintaining a constant UV during radiotherapy is low.

The primary limitation of this study is the retrospective nature. Another limitation is that while the realtime tumor tracking system enables measurements of daily prostatic movement, it does not provide information of rectal volume, the position of seminal vesicles, and other adjacent organs at risk. The correlation between the UV changes and the prostate movement may be negated by the influence of changes in the rectal volume and other factors such as contraction of the pelvic floor muscles. Since rectal stools and flatus were encouraged to be voided before each treatment, and the rectum was degassed when residual gas was identified in the rectum at the time of treatment, the rectal volume was controlled within the routinely possible range. The evaluation of other factors is a task left for future investigations. Here, UV variations could affect the position of the seminal vesicles and other adjacent OAR. Mak et al. reported that bladder and rectal volume changes correlated with seminal vesicle movement especially in the AP direction[26]. If the seminal vesicles also need to be irradiated, it should still be kept in mind that the variation in UV is to be maintained as small.

\section{Conclusions}

The measurements reported here suggest that UV changes have little impact on prostate movement. Attention to UV variation is still needed for patients with prostatic hyperplasia ( $P V \geq 20 \mathrm{ml}$ ) and patients whose seminal vesicle should be irradiated. Further research will be needed to identify other factors that could affect the daily prostate position, and a more ideal preparation protocol ensuring a minimized prostate movement during radiotherapy.

\section{Abbreviations}

$\mathrm{PV}$, prostate volume; UV, urinary volume; LR, left-right; CC, cranial-caudal; $\mathrm{AP}$, anterior-posterior; $\mathrm{Cl}$, confidence interval; RT-CT, treatment planning computed tomography; OAR, organs at risk; and CBCT, cone-beam CT;

\section{Declarations}




\section{- Ethics approval and consent to participate}

This study was approved by the Institutional Review Board of the Hokkaido university hospital.

\section{- Consent for publication}

Not applicable.

- Availability of data and material

The datasets are available from the author on request.

\section{- Competing interests}

There are no conflicts of interest involved in this study.

\section{- Funding}

This work was supported by JSPS KAKENHI Grant Number 18K15535 and 18 K15577.

\section{- Author contributions}

$\mathrm{KN}, \mathrm{TH}$, and SS carried out the treatment planning, participated in the data collection and interpretation, performed the analysis, and participated in the drafting and final revision of the manuscript. TA, TO, RM, NK, RK, KY, and ST participated in the patient care, data collection, and interpretation and in the drafting and final revising of the manuscript. IY participated in the statistical analysis. TY and TY participated in the data analysis and in drafting the manuscript. HA and NS conceived of the study and participated in its design and coordination and helped to draft the manuscript. All authors read and approved the final manuscript.

\section{- Acknowledgements}

We wish to express our appreciation to Kento Gotoh for his contribution to the data collection.

\section{References}

1. Soukup M, Söhn M, Yan D, Liang J, Alber M. Study of robustness of IMPT and IMRT for prostate cancer against organ movement. Int J Radiat Oncol Biol Phys. United States; 2009;75:941-9.

2. Vargas C, Wagner M, Indelicato D, Fryer A, Horne D, Chellini A, et al. Image guidance based on prostate position for prostate cancer proton therapy. Int J Radiat Oncol Biol Phys. United States; 2008;71:1322-8.

3. Lomax AJ. Intensity modulated proton therapy and its sensitivity to treatment uncertainties 2: the potential effects of inter-fraction and inter-field motions. Phys Med Biol. England; 2008;53:1043-56.

4. Pinkawa M, Asadpour B, Gagel B, Piroth MD, Holy R, Eble MJ. Prostate position variability and dosevolume histograms in radiotherapy for prostate cancer with full and empty bladder. Int J Radiat 
Oncol Biol Phys. 2006;64:856-61.

5. Moiseenko V, Liu M, Kristensen S, Gelowitz G, Berthelet E. Effect of bladder filling on doses to prostate and organs at risk: A treatment planning study. J Appl Clin Med Phys. 2007;8:55-68.

6. Nightingale H, Conroy R, Elliott T, Coyle C, Wylie JP, Choudhury A. A national survey of current practices of preparation and management of radical prostate radiotherapy patients during treatment. Radiography. Elsevier Ltd; 2017;23:87-93.

7. Schild SE, Casale HE, Bellefontaine LP. Movements of the prostate due to rectal and bladder distension: implications for radiotherapy. Med Dosim Off J Am Assoc Med Dosim. United States; 1993;18:13-5.

8. Roeske JC, Forman JD, Mesina CF, He T, Pelizzari CA, Fontenla E, et al. Evaluation of changes in the size and location of the prostate, seminal vesicles, bladder, and rectum during a course of external beam radiation therapy. Int J Radiat Oncol Biol Phys. 1995;33:1321-9.

9. Melian E, Mageras GS, Fuks Z, Leibel SA, Niehaus A, Lorant H, et al. Variation in prostate position quantitation and implications for three- dimensional conformal treatment planning. Int J Radiat Oncol Biol Phys. 1997;38:73-81.

10. Zellars RC, Roberson PL, Strawderman M, Zhang D, Sandler HM, Ten Haken RK, et al. Prostate position late in the course of external beam therapy: Patterns and predictors. Int J Radiat Oncol Biol Phys. 2000;47:655-60.

11. Langen KM, Jones DTL. Organ motion and its management. Int J Radiat Oncol Biol Phys. 2001;50:265-78.

12. Pang EPP, Knight K, Hussain A, Fan Q, Baird M, Tan SXF, et al. Reduction of intra-fraction prostate motion - Determining optimal bladder volume and filling for prostate radiotherapy using daily 4D TPUS and CBCT. Tech Innov Patient Support Radiat Oncol. The Authors; 2018;5:9-15.

13. Heng SP, Low SH, Sivamany K. The influence of the bowel and bladder preparation protocol for radiotherapy of prostate cancer using kilo-voltage cone beam CT: Our experience. Indian J Cancer. India; 2015;52:639-44.

14. McNair HA, Wedlake L, Lips IM, Andreyev J, Van Vulpen M, Dearnaley D. A systematic review: Effectiveness of rectal emptying preparation in prostate cancer patients. Pract Radiat Oncol. American Society for Radiation Oncology; 2014;4:437-47.

15. Fujioka C, Ishii K, Yamanaga T, Ogino R, Kishimoto S, Kawamorita R, et al. Optimal bladder volume at treatment planning for prostate cancer patients receiving volumetric modulated arc therapy. Pract Radiat Oncol. American Society for Radiation Oncology; 2016;6:395-401.

16. Grün A, Kawgan-Kagan M, Kaul D, Badakhshi H, Stromberger C, Budach V, et al. Impact of bladder volume on acute genitourinary toxicity in intensity modulated radiotherapy for localized and locally advanced prostate cancer. Strahlentherapie und Onkol. 2019;195:517-25.

17. Stam MR, van Lin ENJT, van der Vight LP, Kaanders JHAM, Visser AG. Bladder filling variation during radiation treatment of prostate cancer: Can the use of a bladder ultrasound scanner and biofeedback optimize bladder filling? Int J Radiat Oncol Biol Phys. 2006;65:371-7. 
18. Moiseenko V, Liu M, Kristensen S, Gelowitz G, Berthelet E. Effect of bladder filling on doses to prostate and organs at risk: A treatment planning study. J Appl Clin Med Phys. 2007;8:55-68.

19. Tsai CL, Wu JK, Wang CW, Hsu FM, Lai MK, Chia-Hsien Cheng J. Using Cone-Beam Computed Tomography to Evaluate the Impact of Bladder Filling Status on Target Position in Prostate Radiotherapy. Strahlentherapie und Onkol. 2009;185:588-95.

20. Gawthrop J, Oates R. Measured bladder volume for radiotherapy of the prostate using the hand-held BladderScan® BVI 3000. J Med Radiat Sci. 2012;59.

21. Janardanan S, Moussa AEM, James P. False positive bladder scan in ascites with anuria. Clin Case Reports. 2019;7:1549-50.

22. Shimizu S, Osaka Y, Shinohara N, Sazawa A, Nishioka K, Suzuki R, et al. Use of implanted markers and interportal adjustment with real-time tracking radiotherapy system to reduce intrafraction prostate motion. Int J Radiat Oncol Biol Phys. 2011;81:e393-9.

23. Homma Y, Gotoh M, Kawauchi A, Kojima Y, Masumori N, Nagai A, et al. Clinical guidelines for male lower urinary tract symptoms and benign prostatic hyperplasia. Int J Urol. 2017;24:716-29.

24. McNair HA, Wedlake L, Lips IM, Andreyev J, Van Vulpen M, Dearnaley D. A systematic review: Effectiveness of rectal emptying preparation in prostate cancer patients. Pract Radiat Oncol. American Society for Radiation Oncology; 2014;4:437-47.

25. Gurjar OP, Arya R, Goyal H. A study on prostate movement and dosimetric variation because of bladder and rectum volumes changes during the course of image-guided radiotherapy in prostate cancer. Prostate Int. Elsevier Ltd; 2020;8:91-7.

26. Mak D, Gill S, Paul R, Stillie A, Haworth A, Kron T, et al. Seminal vesicle interfraction displacement and margins in image guided radiotherapy for prostate cancer. Radiat Oncol. 2012;7:1-9.

\section{Figures}




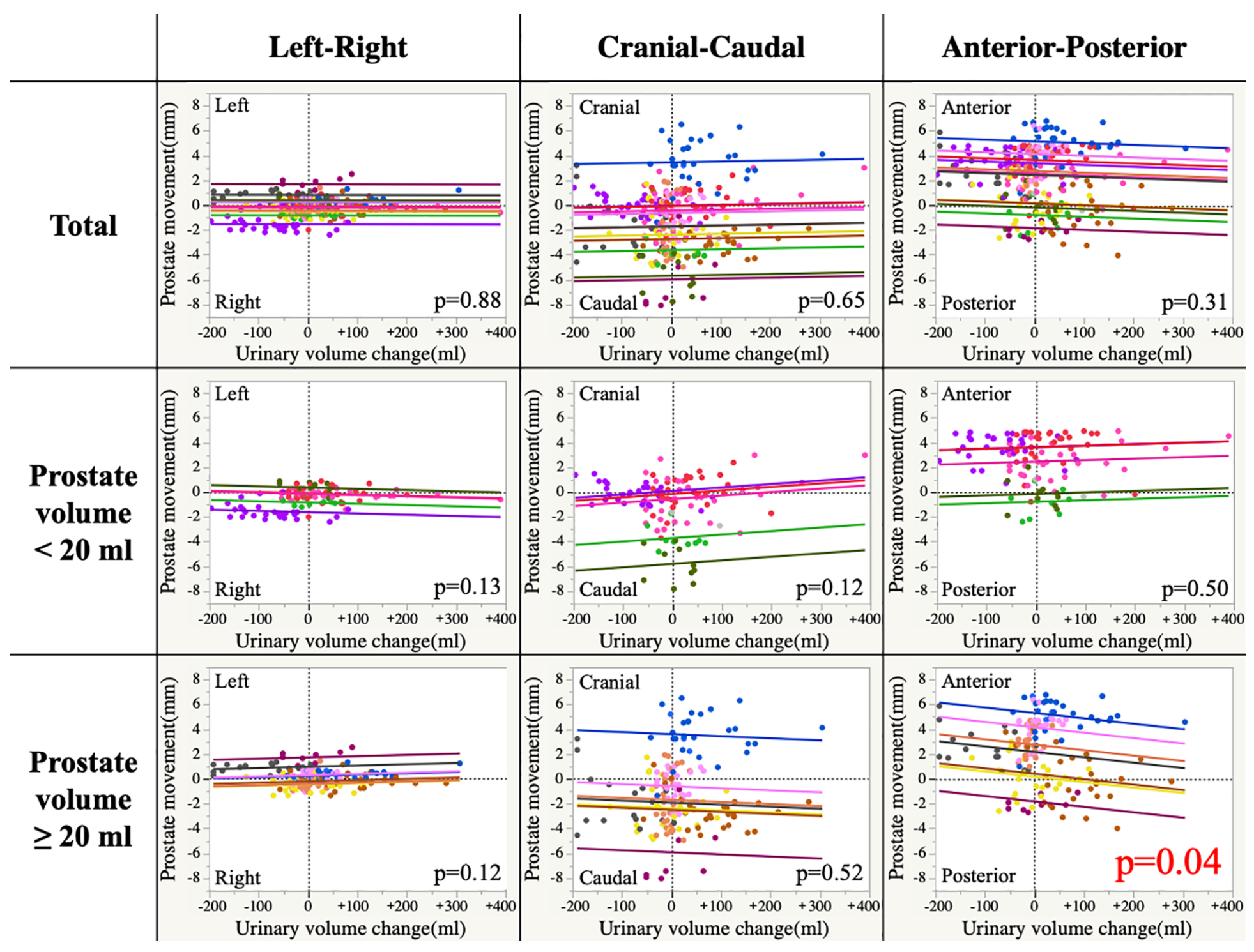

Figure 1

Regression plot graphs of the linear mixed effect model The horizontal axis shows the change in urinary volume from the baseline and the vertical axis shows the movement of the prostate. The color of data points and fitting lines are different for different patients. The positive values of the vertical axis indicate that the prostate moved left, and in the cranial, and anterior directions in the patients, respectively. 


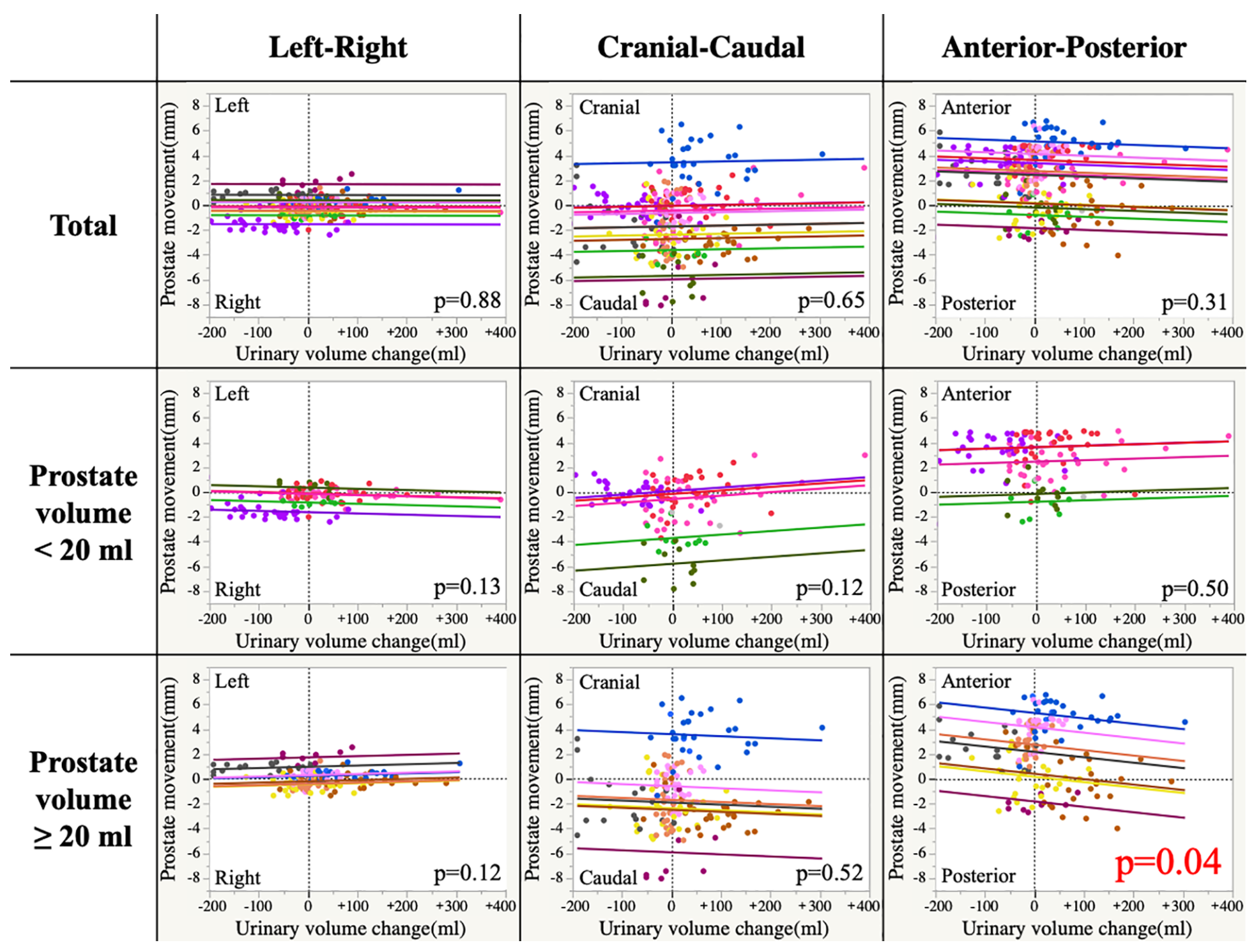

Figure 1

Regression plot graphs of the linear mixed effect model The horizontal axis shows the change in urinary volume from the baseline and the vertical axis shows the movement of the prostate. The color of data points and fitting lines are different for different patients. The positive values of the vertical axis indicate that the prostate moved left, and in the cranial, and anterior directions in the patients, respectively. 


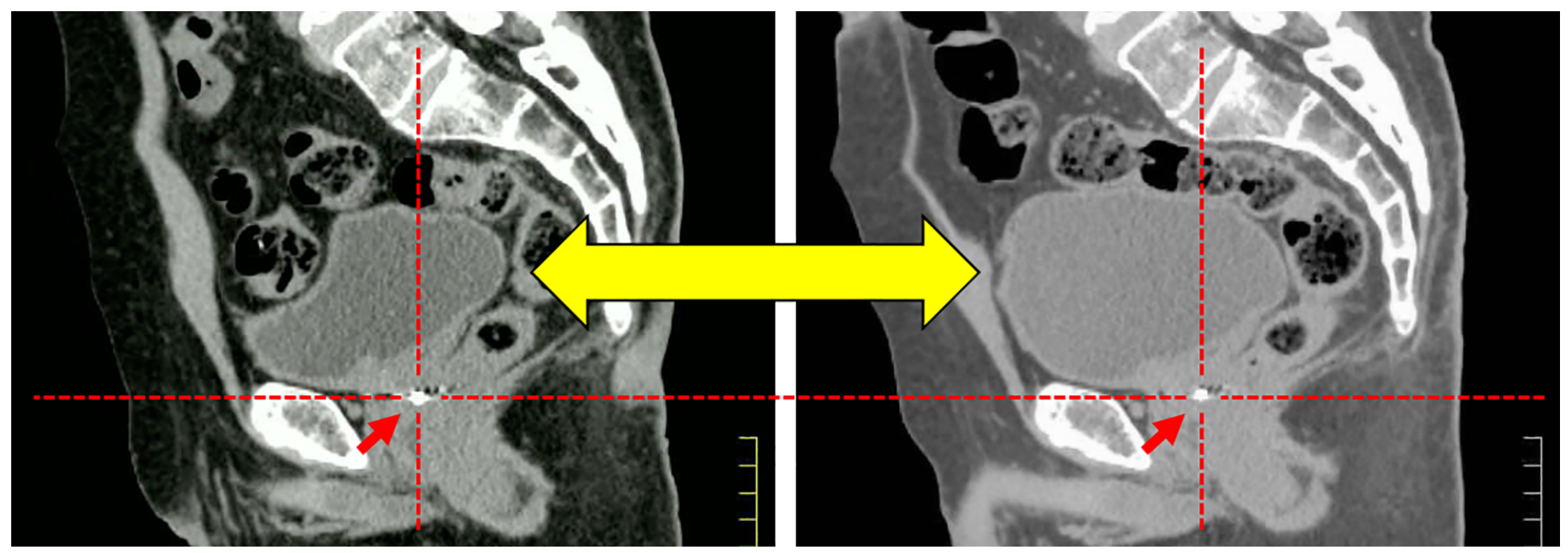

Figure 2

Example of CT images of the same patient with prostate volume $=23.5 \mathrm{ml}$ The red arrows point to the fiducial marker in the prostate. The position of the fiducial marker was largely unchanged despite the large urinary bladder volume change. The urinary volumes in the left and right images were 161.3 and $294.1 \mathrm{ml}$, respectively.

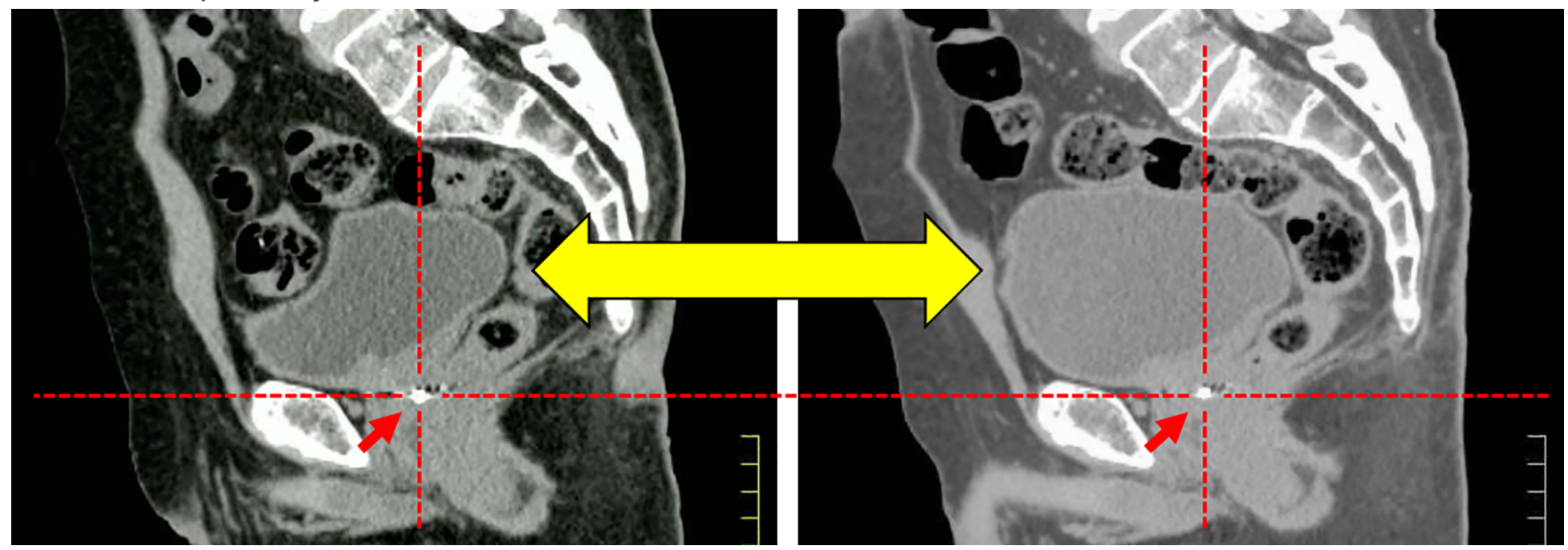

Figure 2

Example of CT images of the same patient with prostate volume $=23.5 \mathrm{ml}$ The red arrows point to the fiducial marker in the prostate. The position of the fiducial marker was largely unchanged despite the large urinary bladder volume change. The urinary volumes in the left and right images were 161.3 and $294.1 \mathrm{ml}$, respectively. 